\title{
Insight into boron-doped diamond Raman spectra characteristic features
}

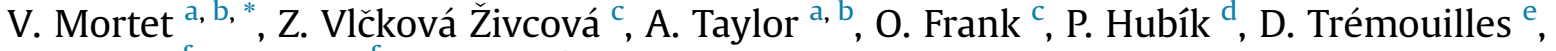 \\ F. Jomard ${ }^{f}$, J. Barjon ${ }^{\mathrm{f}}$, L. Kavan ${ }^{\mathrm{c}}$ \\ a Institute of Physics of the Czech Academy of Sciences, Na Slovance 1999/2, 182 21, Prague 8, Czech Republic \\ ${ }^{\mathrm{b}}$ Czech Technical University in Prague, Faculty of Biomedical Engineering, Sítná 3105, 272 01, Kladno, Czech Republic \\ c J. Heyrovsky Institute of Physical Chemistry of the Czech Academy of Sciences, Dolejškova 3, 182 23, Prague 8, Czech Republic \\ d Institute of Physics of the Czech Academy of Sciences, Cukrovarnická 10, 162 00, Prague 6, Czech Republic \\ e LAAS-CNRS, Université de Toulouse, CNRS, 7, avenue du Colonel Roche, 31031, Toulouse Cedex 4, France \\ ${ }^{\mathrm{f}}$ Groupe d'Etude de la Matière Condensée, Université Versailles St Quentin, 45, avenue des Etats Unis, 78035, Versailles Cedex, France
}

\section{A R T I C L E I N F O}

\section{Article history:}

Received 9 December 2016

Received in revised form

5 January 2017

Accepted 8 January 2017

Available online 9 January 2017

\begin{abstract}
A B S T R A C T
Characteristic Raman features of boron-doped diamond layers with metallic conduction are studied experimentally and discussed in comparison with an extensive selection of literature data. Despite evidence that the main Raman bands are mostly characteristic features of carbon atom vibration modes, their position is found to be proportional to boron concentration. While the origin of the Raman bands located at c.a. $500 \mathrm{~cm}^{-1}$ remains unclear, the band centred at c.a. $1200 \mathrm{~cm}^{-1}$ is attributed to a maximum of phonon density of states due to softening of the Raman wave vector conservation rule. The downshift and broadening of the diamond line are attributed, primarily, to the domain size effect caused by scattering on boron impurities, secondly to the Fano effect due to electronic Raman interaction, and finally to lattice expansion due to the boron doping.
\end{abstract}

() 2017 Elsevier Ltd. All rights reserved.

\section{Introduction}

Boron-doped diamond with metallic conduction is a promising material for various electronic and electrochemical applications. It is a basic building block for the fabrication of ohmic contacts [1] in diamond electronic devices. It is also recognized as one of the best working electrochemical electrodes with high chemical stability, low capacitive current and a large potential window [2]. Diamond electrodes can be used for fabrication of chemical and biological sensors [3], water treatment systems [4], super capacitors [5], dye sensitized solar cells [6-9], etc. Measurement of boron concentration is an important feedback parameter and is usually determined by Secondary Ions Mass Spectroscopy (SIMS) and Hall effect measurements with the assumption that the acceptor concentration is equal to the hole concentration. While Raman spectrum of pure diamond is unmistakably simple, it becomes more and more complex with an increasing concentration of non-diamond carbon

\footnotetext{
* Corresponding author. Institute of Physics of the Czech Academy of Sciences, Na Slovance 1999/2, 182 21, Prague 8, Czech Republic.

E-mail address: mortetv@fzu.cz (V. Mortet).
}

species and other impurities. More specifically, Raman spectra of boron-doped diamond are not fully understood. However, Raman spectroscopy is a simple and non-destructive technique which has been reported to enable the determination of boron concentration [10]. In this work, we analyse characteristic features in Raman spectra of boron-doped diamond with metallic conductivity in order to deepen the understanding of their origin and their dependence with boron concentration.

\section{Experimental}

Epitaxial (111) diamond layers were grown in a $1.5 \mathrm{~kW}$ resonance cavity microwave plasma enhanced chemical vapour deposition system AX5010 from Seki Diamond Systems with a base pressure below $2 \times 10^{-6}$ mbar. Substrates are high-pressure hightemperature synthetic Ib diamond crystals from Sumitomo ELECTRIC Hartmetall GmbH, polished as described in Ref. [11]. Prior to CVD deposition, diamond substrates were cleaned in hot sulphuric acid with potassium nitrate for $10 \mathrm{~min}$, rinsed in hot deionized water, acetone and alcohol in an ultrasonic bath for $10 \mathrm{~min}$. Epitaxial layers were grown at high temperature (c.a. $1000{ }^{\circ} \mathrm{C}$ ) in 
diluted methane in hydrogen $(0.1 \%)$ with the addition of a boron precursor (trimethylborane) with variable boron to carbon $(B / C)$ ratio in the gas phase. The thickness of deposited layers was estimated by double mass measurement using a high precision ME5 microbalance (Sartorius). Electrical properties of deposited layers were measured by the van der Pauw method at room temperature and $100{ }^{\circ} \mathrm{C}$ using a Keithley 6221 current source and Keithley 2182A nano-voltmeter. Secondary-ion mass spectrometry was measured with $\mathrm{Cs}^{+}$primary ions accelerated at $10 \mathrm{keV}$ using a Cameca IMS $4 \mathrm{f}$ apparatus on layers grown with $\mathrm{a} B / \mathrm{C}$ ratio in the gas phase of $20,000 \mathrm{ppm}$ to determine its boron atoms depth distribution. The concentration of boron was quantified using implanted standards. Raman spectroscopy measurements were carried out using a Labram HR spectrometer (Horiba Jobin-Yvon) interfaced to an Olympus microscope (objective $100 \times$ ) equipped with a $50 \mu \mathrm{m}$ confocal hole and a $\mathrm{He}-\mathrm{Ne}$ laser $633 \mathrm{~nm}(1.96 \mathrm{eV})$ with $8 \mathrm{~mW}$ power. The spectrometer was calibrated using the silicon $F_{1 g}$ peak at $520.2 \mathrm{~cm}^{-1}$. Raman spectra were corrected for the spectrometer spectral response.

\section{Results and discussion}

Van der Pauw Hall-effect measurements revealed hole concentrations above the Mott transition concentration (i.e. above $\approx 2-3 \times 10^{20} \mathrm{~cm}^{-3}$ ) [12,13] in all samples. This fact together with the minute variation in resistivity with temperature indicates that all studied layers have metallic type conduction. Deposition rate $\left(r_{t}\right)$, resistivity $(\rho)$, acceptor concentration $\left(N_{a}\right.$, assumed equal to the hole concentration), and mobility $(\mu)$ of deposited layers with variable $\mathrm{B} / \mathrm{C}$ ratio are reported in Table 1 .

Raman spectra of diamond layers deposited with variable $B / C$ ratios, shown in Fig. 1, exhibit the characteristic features of borondoped diamond layers with metallic conduction [14,15]: a wide asymmetric band centred around $500 \mathrm{~cm}^{-1}$ (referred as "L1" in this work), another asymmetric band centred around $1200 \mathrm{~cm}^{-1}$ (referred as "L2"), and the diamond's zone centre phonon line (referred as "RD"), which is redshifted relatively to the intrinsic diamond line $\left(1332.5 \mathrm{~cm}^{-1}\right)$ next to a dip trough (or anti resonant line) centred around 1340-1350 $\mathrm{cm}^{-1}$ (referred as "A"). Even though the occurrence of the L1 and L2 lines appears to be a signature of highly boron-doped diamond, their origin is controversial. Boron and carbon isotopic shifts of the L1 line are not fully consistent to assign this line to boron dimers or boron-carbon vibrations [16]. On the contrary, the carbon isotopic shifts of the L2 line unmistakably point toward a $\mathrm{C}-\mathrm{C}$ vibration mode [16]. The particular shape of the diamond Raman line, or Fano line-shape, has been ascribed to quantum interference between the zone centre optical phonon and a continuum of electronic Raman effect [17]. Finally, the attribution to boron defects of additional low intensity and not well resolved peaks between 500 and $1200 \mathrm{~cm}^{-1}$ [18] are yet to be confirmed. Compared to other spectra, the Raman spectrum of epitaxial layers grown with $\mathrm{B} / \mathrm{C}=2000 \mathrm{ppm}$ does not exhibit the line L1 despite a carrier concentration above the Mott transition and metallic conduction. The absence of line L1 has been

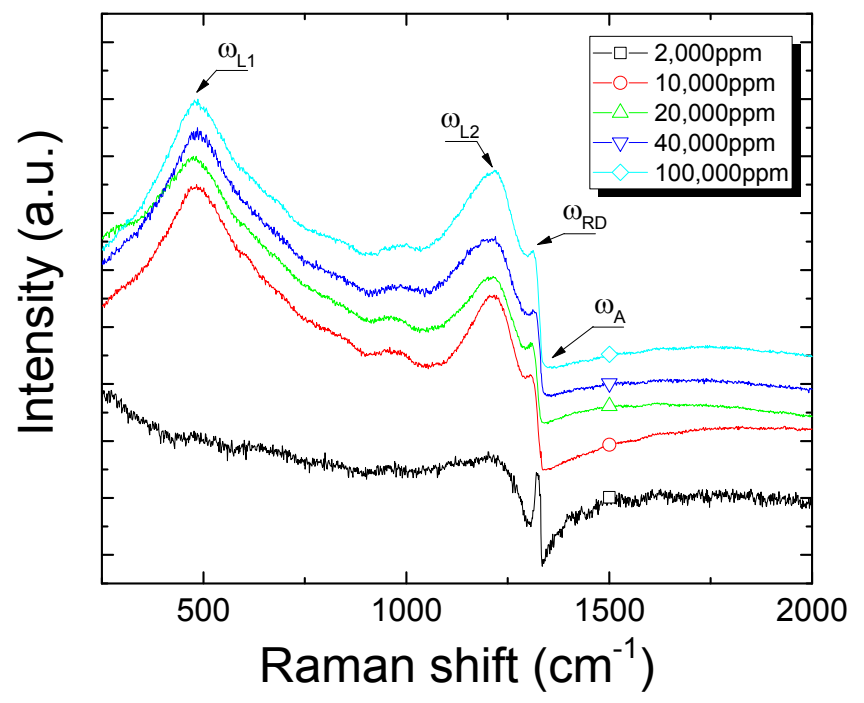

Fig. 1. Raman spectra of epitaxial (111) boron-doped diamond layers grown with various $\mathrm{B} / \mathrm{C}$ ratios in the gas phase. (A colour version of this figure can be viewed online.)

also observed in (100) doped epitaxial diamond layers with metallic conduction grown at a $\mathrm{B} / \mathrm{C}$ ratio of $4000 \mathrm{ppm}$, i.e. higher boron concentration [19].

The Raman shift position of L1 ( $\left.\omega_{\mathrm{L} 1}\right)$ and L2 $\left(\omega_{\mathrm{L} 2}\right)$ lines as a function of the diamond Raman line position $\left(\omega_{\mathrm{RD}}\right)$ are shown on Fig. 2. Despite the scattering of the data attributed to the variability of sample quality, variable excitation wavelengths, variable measurement temperature, and possible stress in the diamond layers [20], the linearity of $\omega_{\mathrm{L} 1}$ and $\omega_{\mathrm{L} 2}$ with $\omega_{\mathrm{RD}}$ is clearly visible. The linear variation of both $\mathrm{L} 2$ and $\mathrm{RD}$ lines with $\mathrm{B} / \mathrm{C}$ ratio of polycrystalline boron-doped diamond reported by S. Ghodbane and A. Deneuville [21] also supports this result. Additionally, the observed linear correlations suggest that both the L1 and L2 lines have the same physical origin as the diamond Raman line and might be a result of vibration modes of the disordered diamond lattice. This is consistent with the assignment of the L2 line to a maximum of the phonon density of states (PDOS) in diamond due to a high defect concentration and softening of the Raman wave vector conservation rule [22]. On the other hand, even though there has not been agreement on the origin of the $\mathrm{L} 1$ peak, i.e. whether it is related to a maximum in PDOS, to boron dimers or boron-to-carbon vibration modes [14], the boron content has been empirically related to the down-shift of the L1 band [10,23].

Fig. 3 shows the variation of the reported boron concentration measured by SIMS ([B] $]_{\text {SIMS }}$ ) as a function of the L1 band maxima $\left(\omega_{\mathrm{L} 1}\right)$. This figure underlines the relatively good linear relationship between boron concentration [B] $]_{\text {SIMS }}$ and L1 line peak position [23]. Linear fitting parameters are reported in Table 2. Considering the aforementioned linear relations between $\omega_{\mathrm{L} 1}, \omega_{\mathrm{L} 2}$ and $\omega_{\mathrm{RD}}$, the boron concentration in diamond is consequently also linear with

Table 1

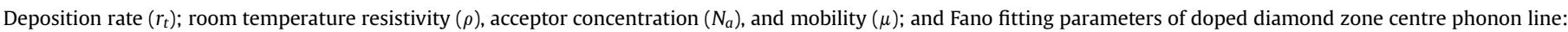
peak frequency $\left(\omega_{0}\right)$ and width $(T)$ and asymmetry parameter $(q)$ as function of B/C ratio in the gas phase during CVD deposition process.

\begin{tabular}{|c|c|c|c|c|c|c|c|}
\hline $\mathrm{B} / \mathrm{C}(\mathrm{ppm})$ & $r_{t}(\mu \mathrm{m} / \mathrm{h})$ & $N_{a}\left(\mathrm{~cm}^{-3}\right)$ & $\rho(\mathrm{m} \Omega \mathrm{cm})$ & $\mu\left(\mathrm{cm}^{2} \mathrm{~V}^{-1} \mathrm{~s}^{-1}\right)$ & $\omega_{0}\left(\mathrm{~cm}^{-1}\right)$ & $\Gamma\left(\mathrm{cm}^{-1}\right)$ & $q$ \\
\hline 2000 & 1.02 & $2.6 \cdot 10^{20}$ & 13 & 1.8 & 1329 & 7.73 & -1.72 \\
\hline 10,000 & 1.14 & $4.6 \cdot 10^{20}$ & 5.8 & 2.3 & 1320 & 17.18 & -1.31 \\
\hline 20,000 & 0.8 & $1.8 \cdot 10^{21}$ & 2.3 & 1.8 & 1320.7 & 15.43 & -1.26 \\
\hline 40,000 & 0.04 & $1.15 \cdot 10^{21}$ & 2.07 & 2.62 & 1323 & 16.25 & -1.75 \\
\hline 100,000 & 0.02 & $2.5 \cdot 10^{21}$ & 0.96 & 2.59 & 1320 & 17.26 & -1.98 \\
\hline
\end{tabular}



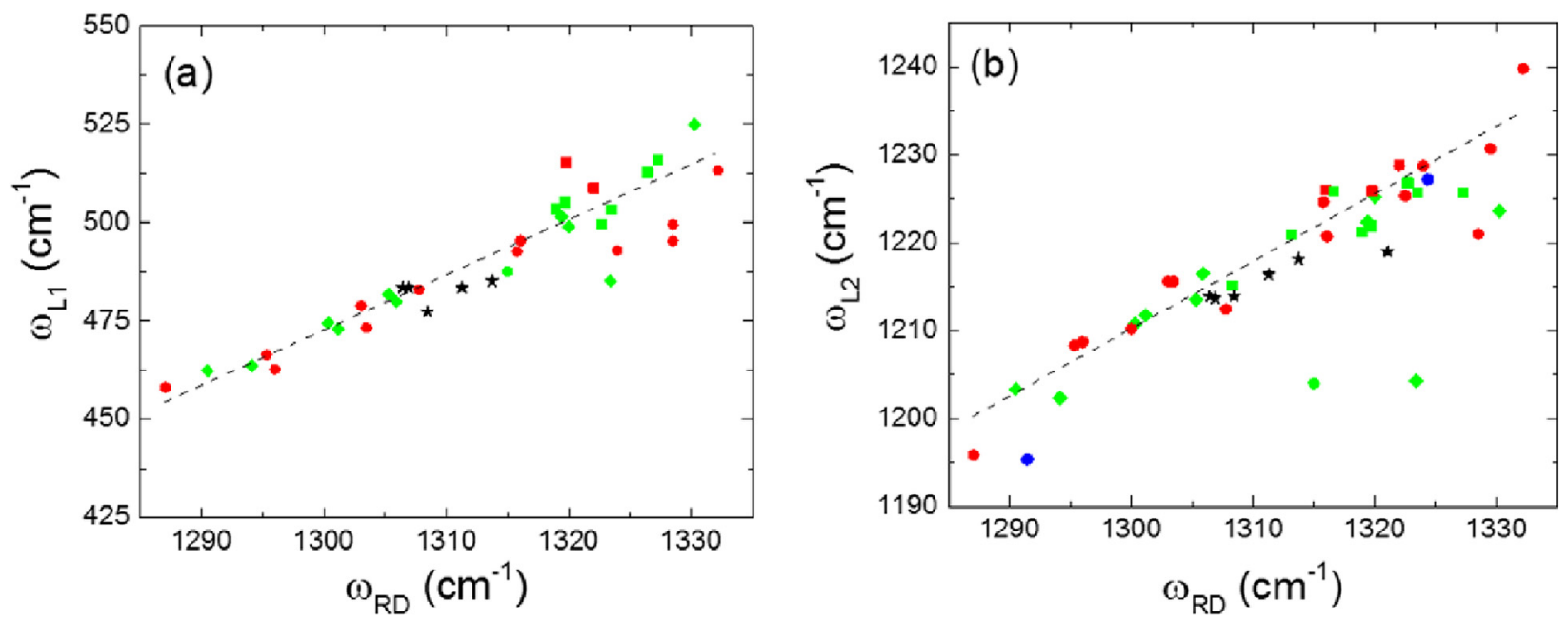

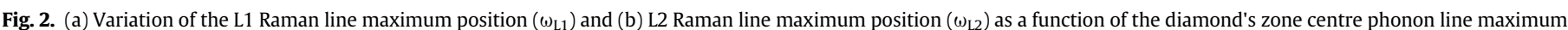

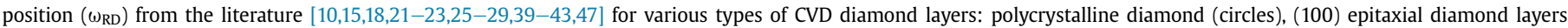

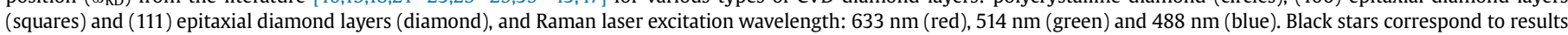
of this study. (A colour version of this figure can be viewed online.)

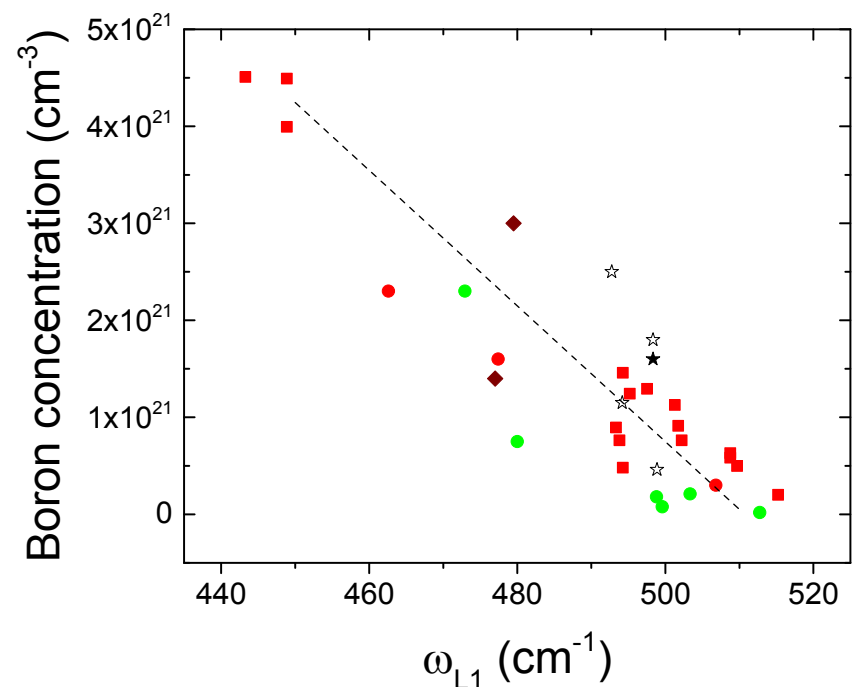

Fig. 3. Boron concentration as measured by SIMS ([B] $]_{\text {SIMS }}$ ) as a function of L1 maximum line $\left(\omega_{\text {L1 }}\right)$ position from the literature $[10,15,23,25,26,39-41]$. Symbols mark various types of CVD diamond layers: polycrystalline diamond (circles), (100) epitaxial diamond layers (squares) and (111) epitaxial diamond layers (diamond), and Raman laser excitation wavelength: $633 \mathrm{~nm}$ (red), $514 \mathrm{~nm}$ (green) and $647 \mathrm{~nm}$ (brown). Black star corresponds to results of this work, full symbol: SIMS measurements, open symbol: Hall effect measurements. (A colour version of this figure can be viewed online.)

the position of the $\mathrm{L} 2$ and $\mathrm{RD}$ lines despite both peaks being clearly identified as pure $\mathrm{C}-\mathrm{C}$ vibration modes. Calculated linear equation coefficients and corresponding zero values, i.e. $\mathrm{X}$ values at $\mathrm{Y}$ equal to zero, are also reported in Table 1. These zero values correspond to the estimated line positions of undoped diamond. The calculated value of the diamond Raman line: $\omega_{\mathrm{RD}}([\mathrm{B}]=0) \sim 1326.9 \mathrm{~cm}^{-1}$ is remarkably very close to the diamond Raman line position. Corrected for the Fano effect (c.a. $4-5 \mathrm{~cm}^{-1}$, see below), this zero value is perfectily consistent with the known position of the intrinsic diamond zone centre phonon line. Similarly, the zero value of the L2 line: $\omega_{\mathrm{L} 2}([\mathrm{~B}]=0) \sim 1230.9 \mathrm{~cm}^{-1}$ is interestingly very close to the diamond phonon density of state maximum located at c.a.1250 $\mathrm{cm}^{-1}$ [20], supporting its assignment to the phonon density of states maximum due to the high concentration of boron defects and the consequent softening of Raman wave-vector conservation rule.

Superimposed onto the L2 line is the diamond line with its characteristic Fano line-shape for boron concentrations higher than the Mott transition. This line-shape results from the interaction of the discrete zone centre phonon Raman line with continuous electronic Raman band and was first reported by Cerdeira, Fjeldly and Cardona in highly doped silicon [24]. In the particular case of constant electronic Raman, the resulting Fano line-shape is analytically described by equation (1).

$F(\varpi)=\frac{(q+\varepsilon)^{2}}{1+\varepsilon^{2}}$

with $\varepsilon(\varpi)=\frac{\varpi-\varpi_{0}}{\Gamma}$

with $\varepsilon(\omega)$ the reduced wavenumber, $q$ is the asymmetry coefficient, $\omega$ is the wavenumber, $\omega_{0}$ and $\Gamma$ are the wavenumber and the full width at half maximum of the phonon's Raman line. The Fano

Table 2

Fitting parameters of linear empiric laws $(\mathrm{Y}=\mathrm{a} \cdot \mathrm{X}+\mathrm{b})$.

\begin{tabular}{|c|c|c|c|c|}
\hline Y (unit) & a (unit) & $X$ (unit) & b (unit) & Zero (unit) \\
\hline$\omega_{\mathrm{L} 1}\left(\mathrm{~cm}^{-1}\right)$ & 1.40 & $\omega_{\mathrm{RD}}\left(\mathrm{cm}^{-1}\right)$ & $-1353.4\left(\mathrm{~cm}^{-1}\right)$ & - \\
\hline$\omega_{\mathrm{L} 2}\left(\mathrm{~cm}^{-1}\right)$ & 0.77 & $\omega_{\mathrm{RD}}\left(\mathrm{cm}^{-1}\right)$ & $212\left(\mathrm{~cm}^{-1}\right)$ & \\
\hline$[\mathrm{B}]_{\text {SIMS }}\left(\mathrm{cm}^{-3}\right)$ & $-6.99 \cdot 10^{19}\left(\mathrm{~cm}^{-2}\right)$ & $\omega_{\mathrm{L} 1}\left(\mathrm{~cm}^{-1}\right)$ & $3.57 \cdot 10^{22}\left(\mathrm{~cm}^{-3}\right)$ & $510.4\left(\mathrm{~cm}^{-1}\right)$ \\
\hline$[\mathrm{B}]_{\text {SIMS }}\left(\mathrm{cm}^{-3}\right)$ & $-1.28 \cdot 10^{20}\left(\mathrm{~cm}^{-2}\right)$ & $\omega_{\mathrm{L} 2}\left(\mathrm{~cm}^{-1}\right)$ & $1.57 \cdot 10^{23}\left(\mathrm{~cm}^{-3}\right)$ & $1230.9\left(\mathrm{~cm}^{-1}\right)$ \\
\hline$[\mathrm{B}]_{\text {SIMS }}\left(\mathrm{cm}^{-3}\right)$ & $-9.81 \cdot 10^{19}\left(\mathrm{~cm}^{-2}\right)$ & $\omega_{\mathrm{RD}}\left(\mathrm{cm}^{-1}\right)$ & $1.30 \cdot 10^{23}\left(\mathrm{~cm}^{-3}\right)$ & $1326.9\left(\mathrm{~cm}^{-1}\right)$ \\
\hline$\Gamma\left(\mathrm{cm}^{-1}\right)$ & -1.29 & $\omega_{0}\left(\mathrm{~cm}^{-1}\right)$ & $1726.6\left(\mathrm{~cm}^{-1}\right)$ & - \\
\hline
\end{tabular}


function possesses two extrema: a resonant peak at $\varepsilon\left(\omega_{R}\right)=-q$ and an anti-resonant peak at $\varepsilon\left(\omega_{\mathrm{A}}\right)=\mathrm{q}^{-1}$. Consequently, a red-shift of the resonant peak corresponds to a negative value of the asymmetric coefficient. Indeed, reported values of asymmetry coefficient in doped diamond are negative with a value c.a. $-2 \pm 0.4$ [22,25-29].

The variation of the Fano line shape with the negative asymmetry coefficient $q$ is illustrated in the supplementary data. Fitting parameters $\left(q, \omega_{0}\right.$ and $\Gamma$ ) of the diamond Fano line-shape can be quickly estimated using a simple analytical method knowing the resonant $\left(\omega_{\mathrm{R}}\right)$, anti-resonant $\left(\omega_{\mathrm{A}}\right)$ and wavenumber at half maximum $\omega_{\mathrm{i}}(1 / 2)$ between $\omega_{\mathrm{R}}$ and $\omega_{\mathrm{A}}$. Estimated fitting parameters of epitaxial layers used in this study are reported in Table 1. Values of the line width $(T)$ are also reported as function of the peak position in Fig. 4 and they are compared to data available from literature. The observed linearity of diamond Raman line broadening and its downward shift (fitting parameters are reported in Table 2) is characteristic of a decrease in domain size and phonon confinement effects. In 1988, Fauchet and Campbell demonstrated that silicon Raman linewidth varies linearly with the frequency shift in microcrystalline silicon with variable domain size [30]. Later, LeGrice et al. reported that the same linear relation applies to diamond [31] based on a comparison of silicon and diamond phonon dispersion curves. However, the determined proportionality between diamond's line maxima and full width at half maximum is c.a. 2.5 times smaller than the one calculated for silicon. Another reason for the downshift of the doped diamond Raman mode is lattice expansion, which inevitably occurs upon doping. Following the study of Wojewoda et al. [32], we estimate the upper limit of the lattice expansion for the maximum doping presented in our data to be $\sim 0.25-0.3 \%$ [32]. With the Grüneisen parameter of the zone centre phonon of $\sim 1$ [33], the maximum Raman shift for $[\mathrm{B}] \sim 5 \times 10^{21} \mathrm{~cm}^{-3}$ reaches $6.6-8 \mathrm{~cm}^{-1}$.

The effect of domain size on full width at half maximum $(\Gamma)$ and position of the diamond Raman line have been studied by several groups of researchers [20,31,34,35]. A noticeable down shift and an inversely proportional line broadening with domain size has been reported by Zaitsev [20] and Yoshikawa [35]. Experimental data

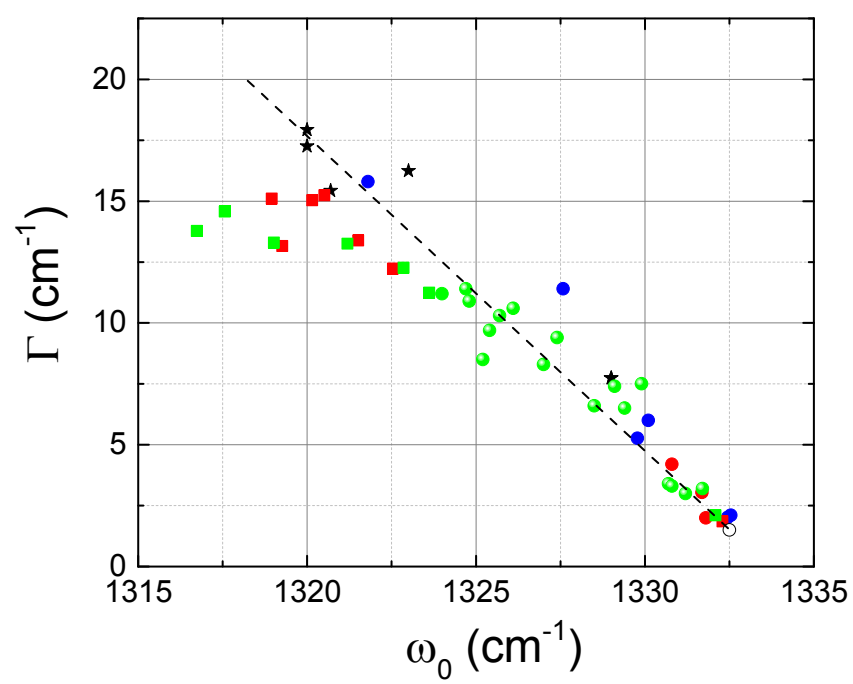

Fig. 4. Variation of fitted diamond's zone centre phonon line width $(\Gamma)$ as a function of fitted line position $\left(\omega_{0}\right)$ using Fano function from various reports $[16,27,28,42-46]$ for various types of CVD diamond layers: polycrystalline diamond (circles), (100) epitaxial diamond layers (squares) and (111) epitaxial diamond layers (diamond), and Raman laser excitation wavelength: $633 \mathrm{~nm}$ (red), $514 \mathrm{~nm}$ (green) and $488 \mathrm{~nm}$ (blue). Black stars correspond to results of this study. (A colour version of this figure can be viewed online.) from Refs. [31,34-36] of domain size $(L)$ as a function of $\Gamma$ of undoped diamond nano-crystals, reported in Fig. 5, more closely follow Zaitsev's model in comparison to Yoshikawa's model. Average boron-to-boron atom distance $\left(d_{B-B}\right)$ is also reported on Fig. 5 as a function of the Fano fitted $\Gamma$, i.e. diamond Raman line width determined from literature. Average boron-to-boron atom distance $\left(d_{B-B}\right)$ is calculated from the boron concentration using:

$d_{B-B}(n m)=10^{7} / \sqrt[3]{[B]\left(\mathrm{cm}^{3}\right)}$

Boron concentration is estimated from the diamond line position using the aforementioned linear relation. Fig. 5 clearly shows that the average boron-to-boron atom distance follows the same variation with the diamond line width as domain size varies, though with a factor of c.a. 5 difference. This result further designates that the diamond line broadening and red-shifting are due to a domain size effect by phonon scattering on boron impurities. This result is not too surprising as impurities are known to strongly reduce semiconductors' thermal conductivity by two effects: carrier-phonon scattering (for electrically active impurities) and phonon-impurity scattering (due to the impurities' mass and radius differences) [37].

In an attempt to conceive the relation between the domain size effect and the boron concentration in doped diamond, we calculated the radial distribution function of boron atoms $\left(\mathrm{RDF}_{\mathrm{B}}\right)$ in doped diamond (see Fig. 6). The $\mathrm{RDF}_{\mathrm{B}}$ has been computed using a supercell of $i^{3}$ ( $i$ being an integer) elementary cubic cells with a randomly positioned boron atom (see Fig. $6 a-b$ ). The size of the elementary cubic cell is arbitrary set to unity but can also be defined as the average boron-to-boron atom distance (a). The boron atom position in each elementary cell was randomly positioned around the cube's centre using a normal distribution with the variance $\sigma^{2}=1 /(2 \pi)$ [38]. The $\mathrm{RDF}_{\mathrm{B}}$ was determined by computing the number of boron atoms as a function of distance $(r)$ to the origin (O) of the supercell and normalized to the surface of the sphere of the same radius. The $\mathrm{RDF}_{\mathrm{B}}$ has been calculated on $1 / 8$ of the space due to the system symmetry. Fig. 6 b shows the doped diamond can be locally seen as a quasi-perfect crystal encapsulated by discontinuous layers of spherical shells with a variable concentration of boron atoms. The first defective spherical shell is located at

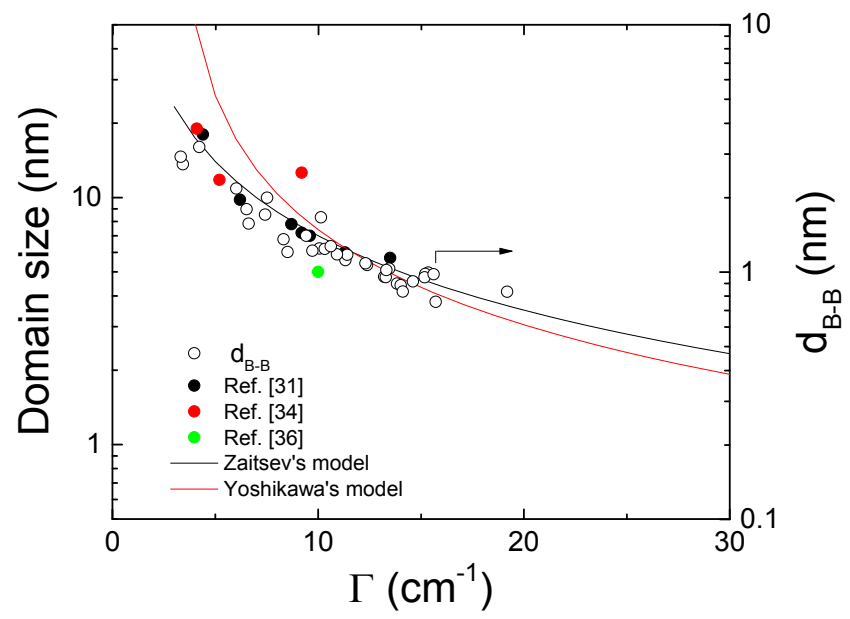

Fig. 5. Diamond domain size (left scale) compared to average boron-to-boron distance (right scale) as a function of zone centre phonon Raman line width. Black line: Zaitsev's model [20]; red line: Yoshikawa's model [35]; full circles: experimental values from Ref. [31] (black) [34], (red) and [35] (green); open circle: average boron-to-boron distance extracted from Refs. [10,22,26-29,43,45] and this work. (A colour version of this figure can be viewed online.) 


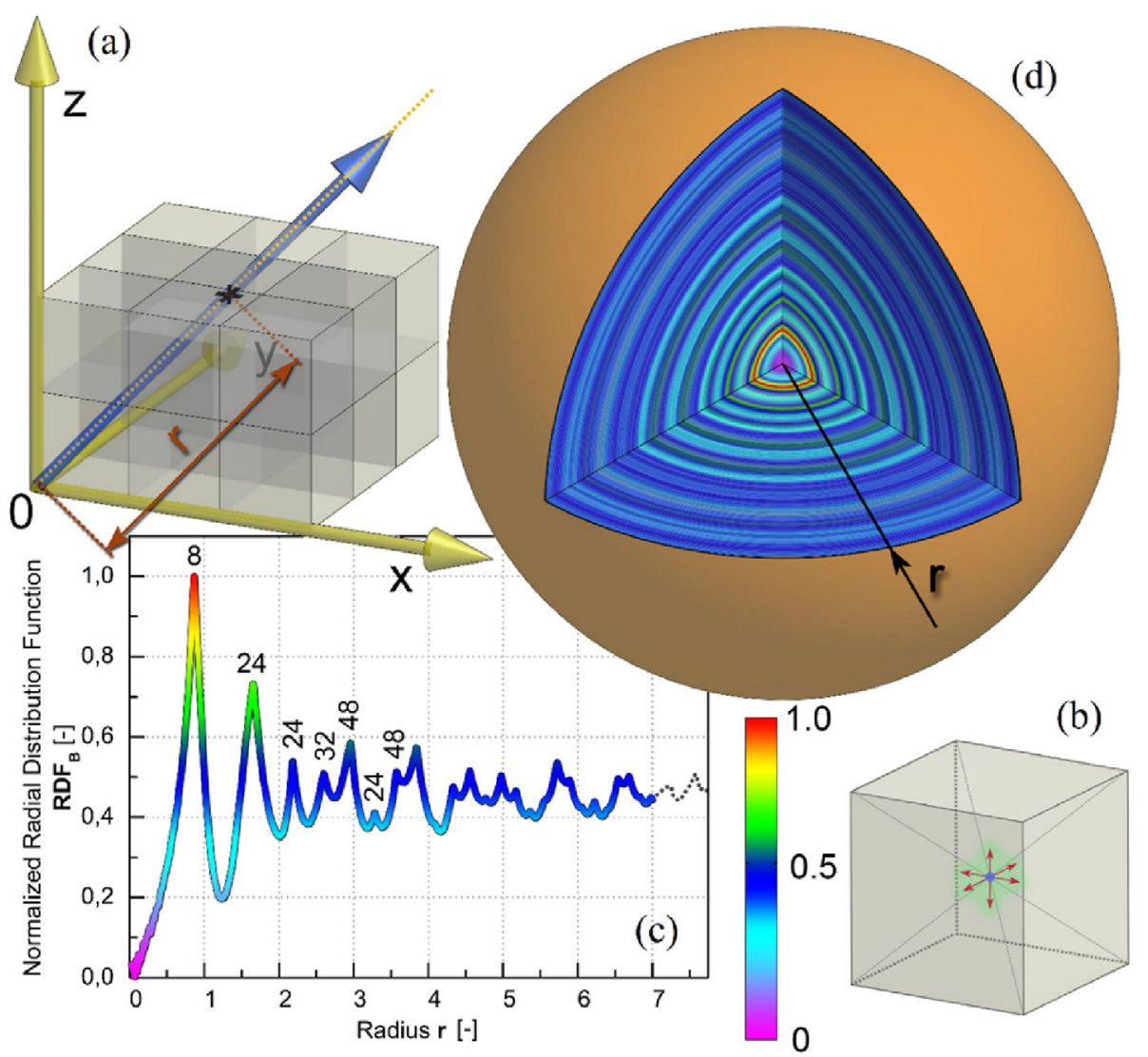

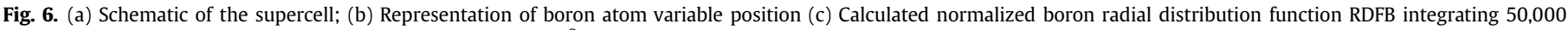

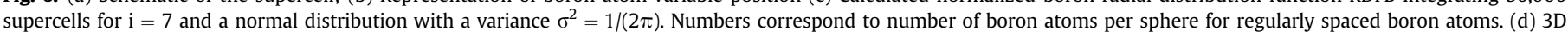
representation of the RDF. (A colour version of this figure can be viewed online.)

$r \sim \sqrt{3} / 2$ with 8 boron atoms. As the radius increases, the distance between consecutive defective spherical shells decreases until their discontinuity vanishes and the defect concentration becomes quasi constant, i.e. the diamond crystal can be locally seen as low defective spherical domains embedded in a continuously defective crystal. The transition between discontinuous and continuous defective spheres is independent of boron concentration and is located at a radius of $2-3$ units, which corresponds to a domain size of 4-6 times the boron-to-boron atom average distance. This value is roughly consistent with the proportionality factor of domain size and boron to boron atom distance. From this novel approach and beside the Fano effect, phonon scattering on randomly distributed boron impurities is the primary effect which broadens and redshifts the Raman zone centre phonon line in boron doped diamond layers comparably as phonon scattering at grain boundaries in nano-diamond particles.

\section{Conclusion}

Epitaxial diamond layers with variable boron concentrations and metallic conductivity were prepared by microwave plasma enhanced chemical vapour deposition and characterized by Raman spectroscopy. Despite evidence that the main Raman bands are mostly characteristic features of carbon atom vibration modes in diamond, their position is proportional to the boron concentration. Analysis of Fano fitting parameters indicates that the downshift and broadening of the diamond line are primarily due to phonon confinement, secondly to electronic Raman interaction and finally to lattice expansion due to the boron doping. Analysis of boron atoms radial distribution function supports this assertion, as it shows that doped diamond can be considered as nanometre sized domains encapsulated in a highly defective matrix.

\section{Acknowledgements}

The work was financially supported by the project $13-31783$ S of Czech Science Foundation, the French-Czech Project Barrande 35785SC of the Czech Ministry of Education, Youth and Sports and the J.E. Purkyne fellowship awarded to V. Mortet by the Czech Academy of Sciences. We also acknowledge Ing. Lucie Holasová and Ing. Jaroslava Hniličková of the Institute of Organic Chemistry and Biochemistry CAS, v.v.i. for mass measurements and Ing. Lukáš Kadeřávek and Ing. Lucie Drbohlavova of Institute of Physics CAS, v.v.i. for their contribution in the preparation of the manuscript.

\section{Appendix A. Supplementary data}

Supplementary data related to this article can be found at http:// dx.doi.org/10.1016/j.carbon.2017.01.022.

\section{References}

[1] L. Gherardini, P. Michaud, M. Panizza, C.H. Comminellis, N. Vasistas, Electrochemical oxidation of 4-chlorophenol for wastewater treatment: definition of normalized current efficiency $(\phi)$, J. Electrochem. Soc. 148 (2001) D78-D82.

[2] A. Fujishima, Y. Einaga, T.N. Rao, D.A. Tryk, Diamond Electrochemistry, Elsevier, Tokyo, 2005.

[3] Y. Einaga, J.S. Foord, G.M. Swain, Diamond electrodes: diversity and maturity, MRS Bull. 39 (2014) 525-532.

[4] B.P. Chaplin, Critical review of electrochemical advanced oxidation processes 
for water treatment applications, Env. Sci. Process. Impact 16 (2014) $1182-1203$.

[5] S. Yu, N.J. Yang, H. Zhuang, J. Meyer, S. Mandal, O.A. Williams, I. Lilge, H. Schonherr, X. Jiang, Electrochemical supercapacitors from diamond, J. Phys. Chem. C 119 (2015) 18918.

[6] H. Krysova, Z. Vlckova-Zivcova, J. Barton, J. Barton, V. Petrak, M. Nesladek, et al., Visible-light sensitization of boron-doped nanocrystalline diamond through non-covalent surface modification, Phys. Chem. Chem. Phys. 17 (2015) 1165-1172.

[7] S.W. Yeap, D. Bevk, X. Liu, H. Krysova, A. Pasquarelli, D. Vanderzande, et al., Diamond functionalization with light-harvesting molecular wires: improved surface coverage by optimized Suzuki cross-coupling conditions, RSC Adv. 4 (2014) 42044-42053.

[8] H. Krysova, L. Kavan, Z. Vlckova-Zivcova, W.S. Yeap, P. Verstappen, W. Maes, K. Haenen, et al., Dye-sensitization of boron-doped diamond foam: champion photoelectrochemical performance of diamond electrodes under solar light illumination, RSC Adv. 5 (2015) 81069-81077.

[9] H. Krysova, J. Barton, V. Petrak, R. Jurok, M. Kuchar, P. Cigler, et al., Efficiency and stability of spectral sensitization of boron-doped-diamond electrodes through covalent anchoring of a donor-acceptor organic chromophore (P1), Phys. Chem. Chem. Phys. 18 (2016) 16444-16450.

[10] M. Bernard, A. Deneuville, P. Muret, Non-destructive determination of the boron concentration of heavily doped metallic diamond thin films from Raman spectroscopy, Diam. Relat. Mater. 13 (2004) 282.

[11] V. Mortet, L. Fekete, P. Ashchleulov, A. Taylor, P. Hubik, D. Tremouille, et al., (100) substrate processing optimization for fabrication of smooth boron doped epitaxial diamond layer by PE CVD, in: Proceedings of NANOCON 2014, 6th International Conference, 2014, pp. 115-119. Brno, Czech Republic, EU.

[12] N.F. Mott, Conduction in Non-crystalline Materials, Oxford University Press, New York, 1987.

[13] Y. Takano, M. Nagao, T. Takenouchi, H. Umezawa, I. Sakaguchi, M. Tachiki, et al., Superconductivity in polycrystalline diamond thin films, Diam. Relat. Mater. 14 (2005) 1936-1938.

[14] M. Bernard, C. Baron, A. Deneuville, About the origin of the low wave number structures of the Raman spectra of heavily boron doped diamond films, Diam. Relat. Mater. 13 (2004) 896-899.

[15] P.W. May, W.J. Ludlow, M. Hannaway, P.J. Heard, J.A. Smith, K.N. Rosser Raman and conductivity studies of boron-doped microcrystalline diamond, facetted nanocrystalline diamond and cauliflower diamond films, Diam. Relat. Mater. 17 (2008) 105-117.

[16] V.A. Sidorov, E.A. Ekimov, Superconductivity in diamond, Diam. Relat. Mater 19 (2010) 351-357.

[17] E. Gheeraert, P. Gonon, A. Deneuville, L. Abello, G. Lucazeau, Effect of boron incorporation on the "quality" of MPCVD diamond films, Diam. Relat. Mater. 2 (1993) 742-745.

[18] M. Mermoux, F. Jomard, C. Tavares, F. Omnes, E. Bustarret, Raman characterization of boron-doped $\{111\}$ homoepitaxial diamond layers, Diam. Relat. Mater. 15 (2006) 572-576.

[19] L. Kavan, Vlčková Živcová, V. Petrák, O. Frank, P. Janda, H. Tarabkova, et al., Boron-doped diamond electrodes: electrochemical, atomic force microscopy and Raman study towards corrosion-modifications at nanoscale, Electrochim. Acta 175 (2015) 626-636.

[20] A.M. Zaitsev, Optical Properties of Diamond, Data Handbook, 2001 edition, Springer, August 9, 2001.

[21] S. Ghodbane, A. Deneuville, Specific features of $325 \mathrm{~nm}$ Raman excitation of heavily boron doped polycrystalline diamond films, Diam. Relat. Mater. 15 (2006) 589-592.

[22] P. Szirmai, T. Pichler, O.A. Williams, S. Mandal, C. Bauerle, F. Simon, A detailed analysis of the Raman spectra in superconducting boron doped nanocrystalline diamond, Phys. Status Solidi B 249 (2012) 2656-2659.

[23] P. Achatz, F. Omnès, L. Ortéga, C. Marcenat, J. Vacík, V. Hnatowicz, et al., Isotopic substitution of boron and carbon in superconducting diamond epilayers grown by MPCVD, Diam. Relat. Mater. 19 (2010) 814-817.

[24] F. Cerdeira, T.A. Fjeldly, M. Cardona, Effect of free carriers on zone-center vibrational modes in heavily doped p -type Si. II. Optical modes, Phys. Rev. 8 (1973) 4734.
[25] K. Ushizawa, K. Watanabe, T. Ando, I. Sakaguchi, M. Nishitani-Gamo, Y. Sato H. Kanda, Boron concentration dependence of Raman spectra on $\{100\}$ and $\{111\}$ facets of B-doped CVD diamond, Diam. Relat. Mater. 7 (1998) 1719-1722.

[26] E. Bustarret, E. Gheeraert, K. Watanabe, Optical and electronic properties of heavily boron-doped homo-epitaxial diamond, Phys. Stat. Sol. (a) 199 (2003) 9-18.

[27] F. Pruvost, A. Deneuville, Analysis of the fano in diamond, Diam. Relat. Mater vol. 10 (2001) 531-535.

[28] C. Piccirillo, A. Mainwood, G. Davies, C.M. Penchina, A. Tajani, M. Bernard, et al., The temperature dependence of the infrared absorption and Raman spectra due to boron in diamond, Phys. Stat. Sol. (a) 193 (2002) 529-534.

[29] P. Gonon, E. Gheeraert, A. Deneuville, F. Fontaine, L. Abello, G. Lucazeau, Characterization of heavily B-doped polycrystalline diamond films using Raman spectroscopy and electron spin resonance, J. Appl. Phys. 78 (1995) 7059-7062.

[30] P.M. Fauchet, I.H. Campbell, Raman spectroscopy of low-dimensional semiconductors, Crit. Rev. Solid State Mater. Sci. 14 (1988) S79-S101.

[31] Y.M. LeGrice, R.J. Nemanich, J.T. Glass, Y.H. Lee, R.A. Rudder, R.J. Markunas, Domain size determination in diamond thin films, Mat. Res. Soc. Symp. Proc. 162 (1990) 219-224.

[32] T. Wojewoda, P. Achatz, L. Ortéga, F. Omnès, C. Marcenat, E. Bourgeois, et al., Doping-induced anisotropic lattice strain in homoepitaxial heavily borondoped diamond, Diam. Relat. Mater. 17 (2008) 1302-1306.

[33] K. Kunc, I. Loa, K. Syassen, Equation of state and phonon frequency calculations of diamond at high pressures, Phys. Rev. B 68 (2003), 094107-094107-9.

[34] C. Johnston, A. Crossley, P.R. Chalker, I.M. Buckley-Golder, K. Kobashi, High temperature Raman studies of diamond thin films, Diam. Relat. Mater. 1 (1992) 450-456.

[35] M. Yoshikawa, Y. Mori, M. Maekawa, G. Katagiri, H. Ishida, A. Ishitani, Raman scattering from diamond particles, Appl. Phys. Lett. 62 (1993) 3114-3116.

[36] S. Prawer, K.W. Nugent, D.N. Jamieson, J.O. Orwa, L.A. Bursill, J.L. Peng, The Raman spectrum of nanocrystalline diamond, Chem. Phys. Lett. 332 (2000) 93-97.

[37] M. Asheghi, K. Kurabayashi, R. Kasnavi, K.E. Goodson, Thermal conduction in doped single-crystal silicon films, J. Appl. Phys. 91 (2002) 5079-5088.

[38] S.M. Stigler, A modest proposal: a new standard for the normal, Am. Stat. 36 (1982) 137-138.

[39] A. Crisci, M. Mermoux, B. Saubat-Marcus, Deep ultra-violet Raman imaging of CVD boron-doped and non-doped diamond films, Diam. Relat. Mater. 17 (2008) 1207.

[40] S. Prawer, R.J. Nemanich, Raman spectroscopy of diamond and doped diamond, Phil. Trans. R. Soc. Lond. A 362 (2004) 2537.

[41] Z. Vlčková Živcová, O. Frank, V. Petrák, H. Tarábková, J. Vacík, M. Nesládek, et al., Electrochemistry and in situ Raman spectroelectrochemistry of low and high quality boron doped diamond layers in aqueous electrolyte solution, Electrochim. Acta 87 (2013) 518-525.

[42] Y.G. Wang, S.P. Lau, B.K. Tay, X.H. Zhang, Resonant Raman scattering studies of Fano-type interference in boron doped diamond, J. Appl. Phys. 92 (2002) 7253-7256.

[43] J.W. Ager III, W. Walukiewicz, M. McCluskey, M.A. Plano, M.I. Landstrass, Fano interference of the Raman phonon in heavily boron-doped diamond films grown by chemical vapor deposition, Appl. Phys. Lett. 66 (1995) 616-618.

[44] M. Nesladek, J.J. Mares, D. Tromson, C. Mer, P. Bergonzo, P. Hubik, et al., Superconductivity and low temperature electrical transport in B-doped CVD nanocrystalline diamond, Sci. Technol. Adv. Mater. 7 (2006) S41-S44.

[45] K. Ushizawa, M.N. Gamo, K. Watanabe, I. Sakaguchi, Y. Sato, T. Ando, Raman spectroscopic study on $\{100\}$ facet of boron-doped chemical-vapour-deposited diamond crystals with Fano line fitting, J. Raman Spectrosc. 30 (1999) 957-961.

[46] A.N. Utyuzh, Y.A. Timofeev, A.V. Rakhmanina, Effect of boron impurity on the Raman spectrum of synthetic diamond, Inorg. Mater. 40 (2004) 926-931.

[47] M. Nesládek, D. Tromson, C. Mer, P. Bergonzo, P. Hubik, J. Mares, Superconductive B-doped nanocrystalline diamond thin films: electrical transport and Raman spectra, Appl. Phys. Lett. 88 (2006), 232111-232111. 